\title{
Time-Domain Homogenization of Windings in 3-D Finite Element Models
}

\author{
Ruth V. Sabariego, Patrick Dular, and Johan Gyselinck
}

\begin{abstract}
This paper deals with the time-domain homogenization of multiturn windings in 3-D finite element (FE) models. An elementary two-dimensional (2-D) FE model allows for the global eddy-current characterization of the winding by means of dimensionless frequency and time-domain coefficients regarding skin and proximity effects. These coefficients are used in the FE model of the complete device. The 3-D homogenization method is validated by considering an axisymmetric 120-turn inductor for which a brute-force 2-D FE model (each turn is finely discretized) provides an accurate reference solution.
\end{abstract}

Index Terms-Eddy currents, skin effect, proximity effect, finite element methods, time domain.

\section{INTRODUCTION}

$\mathbf{M}$ ULTITURN windings in electromagnetic devices may be subjected to considerable skin and proximity effects. Most often these effects are simply ignored in the FE resolution stage and the associated losses are estimated a posteriori. However, the performance of the device under study can be significantly altered by the eddy-current effects, which must be directly included in the FE modeling. The brute-force approach, i.e. finely discretizing each separate turn of the winding, is prohibitively expensive in terms of memory requirements and computation time. Dedicated homogenization methods may thus prove essential. In the frequency domain, they usually amount to the use of complex frequencydependent reluctivity and resistance values, the expression of which is obtained analytically [1], or using an elementary FE model [2].

A more general approach, considering conductors of arbitrary cross-section and packing, is proposed in [3]. The incorporation of this technique in a time-domain 2-D FE model has been dealt with in [4]. In this paper, the homogenization method is extended and applied to a time-domain 3-D FE model. By way of validation we consider a 3-D model of an axisymmetric multiturn inductor, for which a brute-force 2-D FE model provides an accurate reference solution.

\section{ChaRACTERIZATION OF THE WINDING}

A complete eddy-current effect characterization of the winding (conductor cross-section, packing type and fill factor) can be carried out by means of a representative 2-D FE model consisting of a central cell and one or more layers of cells around it [3]. In Fig. 1, such a FE model is shown for the winding that will be considered hereafter, viz a round

Manuscript received June 24, 2007. This work was partly supported by the Belgian Science policy (IAP P6/21) and the Belgian French Community (ARC 03/08-298)

R. V. Sabariego and P. Dular are with the Dept. of Electrical Engineering and Computer Science, Institut Montefiore, University of Liège, Belgium (email: r.sabariego@ulg.ac.be, patrick.dular@ulg.ac.be). P. Dular is with the Belgian National Fund for Scientific Research (F.N.R.S.). J. Gyselinck is with the Dept. of Bio-, Electro- and Mechanical Systems, Université Libre de Bruxelles, Belgium (e-mail: johan.gyselinck@ulb.ac.be). conductor with square packing and fill factor $\lambda$ equal to 0.65 . The reduced frequency $X$ is defined as

$$
X=r / \delta=\sqrt{f} \cdot r \sqrt{\pi \sigma \mu_{0}},
$$

with $r=\sqrt{\mathcal{A}_{c} / \pi}$ the equivalent radius of the conductors $\left(\mathcal{A}_{c}\right.$ surface area), $\delta$ the penetration depth at frequency $f$ or pulsation $\omega=2 \pi f, \sigma$ their conductivity, $\mu_{0}=4 \pi 10^{-7} \mathrm{H} / \mathrm{m}$ their permeability and $\nu_{0}=1 / \mu_{0}$ their reluctivity.
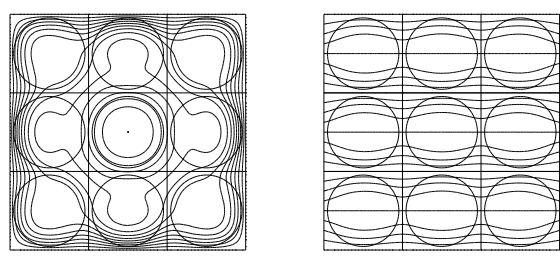

Fig. 1. Elementary FE model with skin- and proximity-effect flux (left and right resp.) - components in phase with $X=2$

Frequency-domain 2-D FE calculations are carried out using the complex notation (symbols in bold, $\boldsymbol{\imath}$ imaginary unit, * indicates conjugate value) for the sinusoidal time variation. The classical magnetic vector potential $(\boldsymbol{a}$-)formulation is adopted. The same net current $\boldsymbol{I}$ is imposed in all conductors by means of electrical circuit equations [5], whereas the average induction $\boldsymbol{b}_{a v}$ in the central cell can be imposed via the boundary conditions [3].

The complex power $\boldsymbol{S}$ (in VA) absorbed by the central cell $\Omega_{c e n}$ is given by [1]

$$
\boldsymbol{S}=P+\imath Q=\frac{l}{2} \int_{\Omega_{c e n}}\left(j^{2} / \sigma+\imath \omega \nu_{0} b^{2}\right) d \Omega,
$$

with $P$ and $Q$ the active and reactive power, and $j^{2} / 2=\boldsymbol{j} \boldsymbol{j}^{*} / 2$ and $b^{2} / 2=\boldsymbol{b} \boldsymbol{b}^{*} / 2$ the r.m.s.-value squared of the current density $\boldsymbol{j}$ and the induction $\boldsymbol{b} ; \boldsymbol{l}$ is the length along the third dimension (which can be arbitrarily taken to be $1 \mathrm{~m}$ ).

The skin- and proximity-effect characterization detailed hereafter amounts to expressing the complex power $S$ in terms of the global quantities $\boldsymbol{I}$ and $\boldsymbol{b}_{a v}$ :

$$
\boldsymbol{S}=\boldsymbol{Z}_{\text {skin }} I^{2} / 2+\boldsymbol{\imath} \omega l \mathcal{A}_{c} \boldsymbol{\nu}_{\text {prox }} b_{a v}^{2} / 2,
$$

thus defining the complex equivalent impedance $\boldsymbol{Z}_{\text {skin }}$ and the complex equivalent reluctivity $\boldsymbol{\nu}_{\text {prox }}$.

\section{A. Skin-effect excitation}

A pure skin-effect excitation is obtained by imposing homogeneous boundary conditions ( $\boldsymbol{a}=0$ on the boundary implies, thanks to symmetry, a zero net flux $\boldsymbol{b}_{a v}=0$ ) and a unit net current $(\boldsymbol{I}=1 \mathrm{~A})$ in all conductors (flux pattern in Fig. 1 left). 
Via the complex power $\boldsymbol{S}$ (2), the complex frequencydependent skin-effect impedance $\boldsymbol{Z}_{\text {skin }}$ and the dimensionless skin-effect coefficients $p_{I}(X)$ and $q_{I}(X)$ are defined [4]:

$$
\boldsymbol{Z}_{\text {skin }}(X)=\frac{P+\boldsymbol{\imath} Q}{\frac{1}{2} I^{2}}=R_{d c}\left(p_{I}(X)+\boldsymbol{\imath} q_{I}(X) \frac{X^{2}}{4}\right),
$$

with $R_{d c}=l /\left(\sigma \mathcal{A}_{c}\right)$ the DC resistance of the conductor.

An approximate dynamic relation between instantaneous voltage $v(t)$ and current $i(t)$ may be obtained for the skineffect impedance $Z_{\text {skin }}(X)$ by considering $n-1$ auxiliary currents $i_{2}(t), i_{3}(t), \ldots$ and a system of $n$ first-order differential equations in terms of the $n$ currents $[I(t)]^{T}=$ $\left[i(t) i_{2}(t) i_{3}(t) \ldots\right]^{T}$ :

$$
[V(t)]=R_{d c}[I(t)]+\left[L^{(n)}\right] \partial_{t}[I(t)],
$$

with $[V(t)]^{T}=\left[\begin{array}{llll}v(t) & 0 & 0 & \ldots\end{array}\right]^{T}$.

The square matrix $\left[L^{(n)}\right]$ is yet to be determined for the winding type at hand. For that purpose the system (5) is rewritten as

$$
[V(t)]=R_{d c}\left([I(t)]+\frac{\sigma \mu_{0} r^{2}}{8 \lambda}\left[\mathcal{S}^{(n)}\right] \partial_{t}[I(t)]\right),
$$

where $\left[\mathcal{S}^{(n)}\right]=\frac{\lambda \nu_{0}}{\sigma r^{2} R_{d c}}\left[L^{(n)}\right]$ is dimensionless [4].

Converting (6) to the frequency domain and calculating $\boldsymbol{v} / \boldsymbol{i}$, the following skin-effect impedance is obtained:

$$
\boldsymbol{Z}_{\text {skin }}^{(n)}(X)=\frac{\boldsymbol{v}}{\boldsymbol{i}}=R_{d c}\left(\left[1^{(n)}\right]+\boldsymbol{\imath} \frac{X^{2}}{4 \lambda}\left[\mathcal{S}^{(n)}\right]\right)_{(1,1)}^{-1},
$$

where $\left[1^{(n)}\right]$ is the $n \times n$ identity matrix and the subscript $(1,1)$ refers to the first diagonal element of the inverse matrix.

For achieving a sufficient agreement between $Z_{\text {skin }}(X)$ and $Z_{\text {skin }}^{(n)}(X)$ in the relevant frequency range $\left[0, X_{\max }\right]$, the parameter $n$ has to be chosen big enough and the matrix $\left[\mathcal{S}^{(n)}\right]$ fitted subsequently. Practice shows that the fitting is straightforward with a symmetric and tridiagonal matrix. For instance, for the considered winding type (see Fig. 1), Fig. 2 evidences the excellent agreement between $Z_{\text {skin }}(X)$ and $Z_{\text {skin }}^{(3)}(X)$ with $n=3, X_{\max }=4$ and the following matrix

$$
\left[\mathcal{S}^{(3)}\right]=\left[\begin{array}{ccc}
1.7968 & 0.6051 & 0 \\
0.6051 & 0.5584 & 0.1874 \\
0 & 0.1874 & 0.4353
\end{array}\right]
$$

The approximations for $n=1$ and $n=2$ are depicted as well.

\section{B. Proximity-effect excitation}

By imposing a unit horizontal (or vertical) induction $\left(\boldsymbol{b}_{a v}=\right.$ $1 \mathrm{~T})$ through appropriate boundary conditions and a zero net current $(\boldsymbol{I}=0)$ in the conductors of the elementary FE model, a pure proximity-effect excitation of the central cell is effected (flux pattern in Fig. 1 right).

Analogously, via the complex power $S$ (2), the complex proximity-effect reluctivity $\boldsymbol{\nu}_{\text {prox }}(X)$ and the dimensionless proximity-effect coefficients $p_{B}(X)$ and $q_{B}(X)$ are defined:

$$
\boldsymbol{\nu}_{\text {prox }}(X)=\frac{Q+\boldsymbol{\imath} P}{\frac{1}{2} \omega l \mathcal{A}_{c} b_{a v}^{2}}=\nu_{0}\left(q_{B}(X)+\boldsymbol{\imath} p_{B}(X) \frac{\lambda X^{2}}{2}\right)
$$

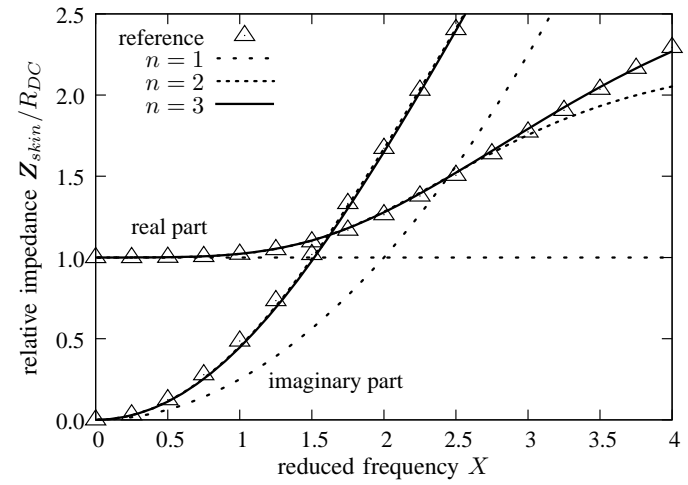

Fig. 2. Relative skin-effect impedance (real $\&$ imaginary parts) vs $X$. The skin-effect impedance (7) $(n=1,2,3)$ is compared to the reference (4)

where factor $\frac{\lambda X^{2}}{2}$ in (9) follows from the analytical expression for low-frequency proximity losses in a round conductor [2].

In the time domain, we have a dynamic relation between the instantaneous magnetic field $h(t)$ and magnetic induction $b(t)$ by considering $n-1$ auxiliary induction components $[B(t)]^{T}=\left[\begin{array}{llll}b(t) & b_{2}(t) & b_{3}(t) & \ldots\end{array}\right]^{T}$. The proximity-effect counterparts of (6-7) are thus given by [4]:

$$
\begin{gathered}
{[H(t)]=\nu_{0}\left([B(t)]+\frac{\lambda \sigma \mu_{0} r^{2}}{4}\left[\mathcal{P}^{(n)}\right] \partial_{t}[B(t)]\right),} \\
\boldsymbol{\nu}_{\text {prox }}^{(n)}(X)=\frac{\boldsymbol{h}}{\boldsymbol{b}}=\nu_{0}\left(\left[1^{(n)}\right]+\boldsymbol{\imath} \frac{\lambda X^{2}}{2}\left[\mathcal{P}^{(n)}\right]\right)_{(1,1)}^{-1},
\end{gathered}
$$

with $[H(t)]^{T}=[h(t) \quad 0 \quad 0 \quad \ldots .]^{T}$ and where the symmetric and tridiagonal matrix $\left[\mathcal{P}^{(n)}\right]$ has to be fitted on the basis of $\boldsymbol{\nu}_{\text {prox }}(X)$.

The relative proximity-effect reluctivity $\boldsymbol{\nu}_{\text {prox }} / \nu_{0}$ is represented in Fig. 3 for the approximation $\nu_{\text {prox }}^{(n)}(X)$ (11) with $n=1,2,3$ and the reference $\boldsymbol{\nu}_{\text {prox }}(X)(9)$. With $n=3$ and the matrix

$$
\left[\mathcal{P}^{(3)}\right]=\left[\begin{array}{ccc}
0.9958 & 0.7197 & 0 \\
0.7197 & 0.5913 & -0.0930 \\
0 & -0.0930 & 0.1489
\end{array}\right]
$$

the approximation is clearly valid beyond $X=4$.

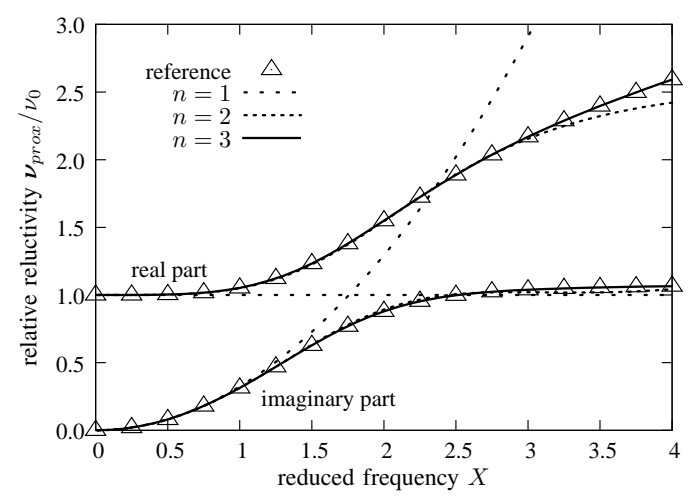

Fig. 3. Relative proximity-effect reluctivity (real \& imaginary parts) vs $X$. Comparison of the proximity-effect reluctivity (11) $(n=1,2,3)$ with reference (9)

From (10), the expressions for the instantaneous magnetic energy density $w_{\text {prox }}^{(n)}$ and joule loss density $p_{\text {prox }}^{(n)}$ can be 
written as

$$
\begin{aligned}
& w_{\text {prox }}^{(n)}(t)=\frac{1}{2} \nu_{0}[B(t)]^{T}[B(t)] \\
& p_{\text {prox }}^{(n)}(t)=\frac{\lambda \sigma r^{2}}{4} \partial_{t}[B(t)]^{T}\left[\mathcal{P}^{(n)}\right] \partial_{t}[B(t)] .
\end{aligned}
$$

\section{Homogenization OF WINDINGS IN 3-D FE MODELS}

We adopt the $\underline{a}-v$ magnetodynamic formulation in a bounded domain $\bar{\Omega}=\Omega_{c} \cup \Omega_{c}^{C}$, with $\Omega_{c}$ conducting and $\Omega_{c}^{C}$ non-conducting (vectors denoted by underlined symbols). The electric field $\underline{e}$ in $\Omega_{c}$ is expressed in terms of a magnetic vector potential $\underline{a}$ and an electric scalar potential $v$ as

$$
\underline{e}=-\partial_{t} \underline{a}-\operatorname{grad} v \text { in } \Omega_{c}, \quad \underline{b}=\operatorname{curl} \underline{a} \text { in } \Omega,
$$

so that the Faraday equation, curl $\underline{e}=-\partial_{t} \underline{b}$, is satisfied. The current density $j_{s}$ in the source domain $\Omega_{s}$ is given. The weak form of the Ampère law, curl $\underline{h}=\underline{j}$, then reads:

$$
\begin{aligned}
\left(\nu \operatorname{curl} \underline{a}, \operatorname{curl} \underline{a}^{\prime}\right)_{\Omega}+ & \left(\sigma \partial_{t} \underline{a}, \underline{a}^{\prime}\right)_{\Omega_{c}}+\left(\sigma \operatorname{grad} v, \underline{a}^{\prime}\right)_{\Omega_{c}} \\
= & \left(\underline{j}_{s}, \underline{a}^{\prime}\right)_{\Omega_{s}}, \quad \forall \underline{a}^{\prime} \in F_{a}(\Omega),
\end{aligned}
$$

where $(\cdot, \cdot)_{\Omega}$ denotes a volume integral in $\Omega$ of the product of its arguments; $F_{a}(\Omega)$ is the gauged function space defined on $\Omega$ and containing the basis functions for $\underline{a}$ and the test function $\underline{a}^{\prime}$. At the discrete level, this space is built with edge finite elements [6].

We consider a multiturn winding in $\Omega$. The axial component of the current due to the small but finite thread of the coil is neglected. In general, the winding is connected to other conductors in the FE model and/or lumped electrical circuit components, and the current flowing through its turns $i(t)$ is not known a priori [6]. For the sake of brevity, a given current $i(t)$ is considered here. For accurately accounting for skin and proximity effects in a brute-force manner, the winding (i.e. conducting material) is treated as part of $\Omega_{c}$. If the winding has a large number of turns, a much more pragmatic and commonly adopted approach consists in homogenizing it. The winding domain (i.e. conducting material plus insulation, now) is then modeled as a so-called stranded conductor in $\Omega_{s} \subset \Omega_{c}^{C}$, carrying the homogenized current density (proportional to $\lambda i$ ). At low frequencies $(X<1)$, this trivial homogenization approach is sufficient. At higher frequencies, further steps are required.

\section{A. Frequency domain}

In case of voltage supply or electrical circuit coupling of the winding, the skin effect is taken into account by simply replacing the DC winding resistance $R_{d c}$ in the electrical circuit equations by the complex impedance $Z_{\text {skin }}(X)$.

The proximity effect is accounted for by adopting in the homogenized winding volume the complex reluctivity $\boldsymbol{\nu}_{\text {prox }}(X)$ for the two perpendicular directions in the plane of the conductor cross-section. In the third direction, the reluctivity $\nu_{0}$ remains unchanged.

\section{B. Time domain}

Skin effect is taken into account by means of the auxiliary currents $\underline{i}_{2}(t), \underline{i}_{3}(t), \ldots$ and the system of differential equations (6) with the fitted matrix $\left[\mathcal{S}^{(n)}\right]$.
As for the proximity effect, we rewrite (10) considering the magnetic vector potential $\underline{a}$ for the average induction $\underline{b}=\underline{b}_{1}$ :

$$
\begin{aligned}
& \underline{h}=\nu_{0} \operatorname{curl} \underline{a}+\frac{\lambda \sigma r^{2}}{4}\left(p_{11} \partial_{t} \operatorname{curl} \underline{a}+\sum_{i=2}^{n} p_{1 i} \partial_{t} \underline{b}_{i}\right), \\
& 0=\nu_{0} \underline{b}_{j}+\frac{\lambda \sigma r^{2}}{4}\left(p_{j 1} \partial_{t} \operatorname{curl} \underline{a}+\sum_{i=2}^{n} p_{j i} \partial_{t} \underline{b}_{i}\right),
\end{aligned}
$$

with $2 \leq j \leq n$ and $p_{i j}$ the fitted elements of $\left[\mathcal{P}^{(n)}\right]$. If $n \geq$ 2 , this implies the introduction of $n-1$ additional induction vector unknowns in the winding region $\Omega_{s}$.

These auxiliary vector fields $\underline{b}_{i}$ must have a zero component perpendicular to the local cross-section of the turns. In practice, we can e.g. adopt nodal basis functions multiplied by two unit vectors that force them in two perpendicular directions in the plane of the cross-section.

Considering (17), the weak form (16) becomes

$\left(\nu \operatorname{curl} \underline{a}, \operatorname{curl} \underline{a}^{\prime}\right)_{\Omega}+\left(\sigma \partial_{t} \underline{a}, \underline{a}^{\prime}\right)_{\Omega_{c}}+\left(\sigma \operatorname{grad} v, \underline{a}^{\prime}\right)_{\Omega_{c}}$ $+\left(\frac{\lambda \sigma r^{2}}{4} p_{11} \partial_{t} \operatorname{curl} \underline{a}, \operatorname{curl} \underline{a}^{\prime}\right)_{\Omega_{s}}+\sum_{i=2}^{n}\left(\frac{\lambda \sigma r^{2}}{4} p_{1 i} \partial_{t} \underline{b}_{i}, \operatorname{curl} \underline{a}^{\prime}\right)_{\Omega_{s}}$

$$
=\left(\underline{j}_{s}, \underline{a}^{\prime}\right)_{\Omega_{s}}, \quad \forall \underline{a}^{\prime} \in F_{a}(\Omega) .
$$

The expression (18) brings about the following weak forms:

$$
\begin{aligned}
& \left(\nu_{0} \underline{b}_{j}, \underline{b}_{j}^{\prime}\right)_{\Omega_{s}}+\left(\frac{\lambda \sigma r^{2}}{4} p_{j 1} \partial_{t} \operatorname{curl} \underline{a}, \underline{b}_{j}^{\prime}\right)_{\Omega_{s}} \\
& +\sum_{i=2}^{n}\left(\frac{\lambda \sigma r^{2}}{4} p_{j i} \partial_{t} \underline{b}_{i}, \underline{b}_{j}^{\prime}\right)_{\Omega_{s}}=0, \quad \forall \underline{b}_{j}^{\prime} \in F_{b}\left(\Omega_{s}\right),
\end{aligned}
$$

with $2 \leq j \leq n . F_{b}\left(\Omega_{s}\right)$ is the function space defined on $\Omega_{s}$ and containing the basis and test functions for $\underline{b}_{i}$ and $\underline{b}_{i}^{\prime}$.

The $2(n-1)$ nodal values associated with each node situated in or on the boundary of $\Omega_{s}$ are the additional unknowns.

\section{ApplicAtion EXAMPLE}

The 3-D homogenization method is applied to an axisymmetric 120-turn inductor with round conductors $\left(\mathcal{A}_{c}=1 \mathrm{~mm}^{2}\right.$, $\left.\sigma=60 \mathrm{MS} / \mathrm{m}, R_{d c}=0.19 \Omega\right)$ and square packing $(\lambda=0.65)$. The magnetic core $\left(\mu_{r}=1000\right)$ has a $3 \mathrm{~mm}$ central air-gap. We assume the core is non-conducting $(\sigma=0)$, which is valid as long as the related magnetic diffusion time is much smaller than the period of interest [7].

A 2-D axisymmetric FE fine model of the inductor is considered in order to validate the time-domain homogenized model (Fig. 4 left). Indeed, this model allows to discretize separately and finely each turn of the winding. It is cumbersome but feasible. In a 3-D FE model of the inductor, however, keeping the same level of refinement is completely out of the question. Taking account of skin and proximity effects requires thus the use of a homogenized model (Fig. 4 right).

Some flux patterns obtained with the 2-D FE fine model are depicted in Fig. 5. One clearly sees the effects of the eddy currents $(X=2$ vs $X=0.25)$ and the air-gap on the flux lines in the winding domain.

We compare global frequency and time-domain quantities related to the energy and the power given by (2) for the axisymmetric 2-D FE fine model and by (3), (13) and (14) for the 3-D FE homogenized model.

The equivalent resistance $R(X)$ and inductance $L(X)$ of the winding are derived from frequency-domain calculations with 

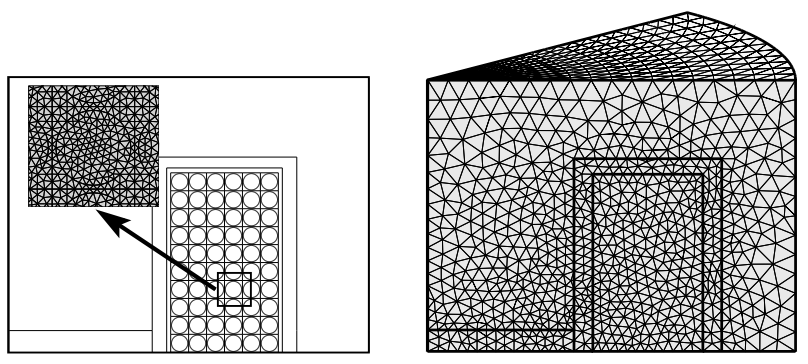

Fig. 4. 2-D FE model of axisymmetric inductor (half height) - detail of the mesh (left). 3-D homogenized FE model - one sixteenth of geometry (right)

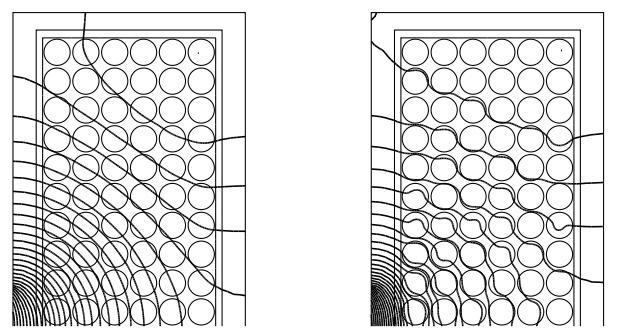

Fig. 5. Flux lines in winding domain for $X=0.25$ (left) and $X=2$ (right)

both models. An excellent agreement is observed in Fig. 6 . Mainly due to the proximity effect, the eddy-current losses increase substantially with frequency, whereas the inductance decreases slightly.
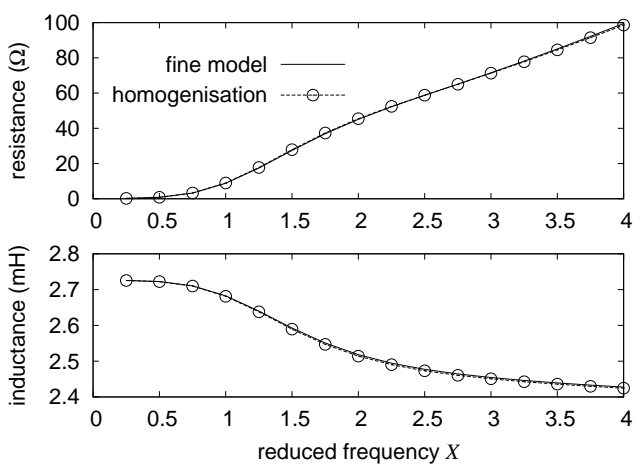

Fig. 6. Equivalent resistance and inductance versus reduced frequency $X$

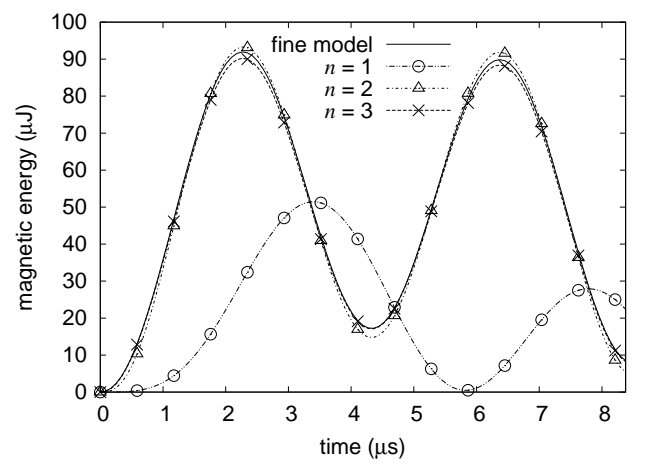

Fig. 7. Magnetic energy in the winding domain versus time obtained with both the fine model and the 3-D homogenized model $(n=1,2,3)$

Time-stepping simulations with imposed sinusoidal current of fundamental frequency $X=3$ (fundamental period $T=$ $1 / f=8.37 \mu \mathrm{s})$ are carried out. The magnetic energy and the

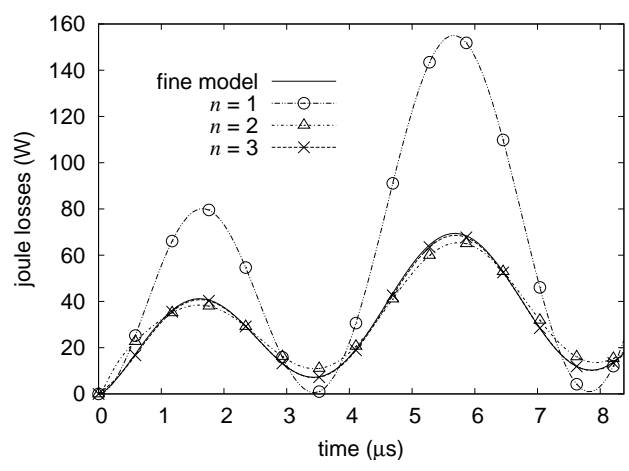

Fig. 8. Joule losses versus time obtained with both the fine model and the 3-D homogenized model $(n=1,2,3)$

total joule losses in the winding as a function of time (one period, time step $\Delta t=T / 100$ ) are shown in Figs. 7 and 8. As expected from Figs. 2 and $3, n=1$ is not enough, additional induction vector unknowns are necessary. The accuracy of the homogenization becomes excellent with $n=3$.

With the 3-D homogenized model and $n=3$, one time step takes $40 \mathrm{~s}$ (59569 unknowns) with a direct LU solver on a Pentium M, 2.28GHz. A brute-force 3-D model would require a much finer mesh (average element size in the winding $\leq$ $\delta / 3$ ), what would be beyond practical limits. This is a fortiori the case when no symmetry can be exploited.

\section{CONCLUSions}

The proposed time-domain homogenization method allows accounting for the skin and proximity effects in a 3-D FE model of a winding with high precision and at an affordable computational cost. Its application, though validated with an axisymmetric test case, is general.

An elementary and computationally cheap 2-D FE model is first used to characterize the winding type by four dimensionless frequency-dependent coefficients. In the time domain, a small symmetric and tridiagonal matrix must be fitted.

In the frequency domain, the coefficients are directly used in the FE model by adopting complex and frequency-dependent values for the resistance and the reluctivity. The 3-D timedomain extension requires a limited number of additional unknowns in the plane of the conductor cross-section.

\section{REFERENCES}

[1] O. Moreau, L. Popiel and J. L. Pages, "Proximity losses computation with a 2D complex permeability modelling," IEEE Trans. on Magn., vol. 34, no. 5, pp. 3616-3619, Sep. 1998.

[2] A. Podoltsev, B. Lebedev, "Analysis of effective resistance and eddycurrent losses in multiturn winding of high-frequency magnetic components," IEEE Trans. on Magn., vol. 39, no. 1, pp. 539-548, Jan. 2003.

[3] J. Gyselinck and P. Dular, "Frequency-domain homogenization of bundles of wires in 2-D magnetodynamic FE calculations," IEEE Trans. on Magn., vol. 41, no. 5, pp. 1416-1419, May 2005.

[4] J. Gyselinck, R. V. Sabariego and P. Dular, "Time-domain homogenization of windings in 2-D finite element models," IEEE Trans. on Magn., vol. 43, no. 4, pp. 1297-1300, Apr. 2007.

[5] P. Lombard, G. Meunier, "A general purpose method for electric and magnetic combined problems for $2 \mathrm{D}$, axisymmetric and transient systems," IEEE Trans. on Magn., vol. 29, no. 2, pp. 1737-1740, 1993.

[6] P. Dular, F. Henrotte, W. Legros, "A general and natural method to define circuit relations associated with magnetic vector potential formulations," IEEE Trans. on Magn., vol. 35, no. 3, pp. 1630-1633, May 1999.

[7] J. R. Brauer, Magnetic actuators and sensors, Wiley IEEE Press, 2006. 